\title{
Achromatic Colouring of the Central Graph of Some Specialgraphs
}

\author{
K.P.Thilagavathy, A.Santha, G.S. Nandakumar
}

\begin{abstract}
In this research investigation, the achromatic number of central graph of double wheel graph, wind mill graph andn- anti prism graph have been studied. In addition the structural properties of these graphs have also been studied.
\end{abstract}

Key Words: Double wheel graph, Wind mill graph, Anti prism graph, achromatic number, b-chromatic number, Central graph Mathematics subject classification: $05 C 15$

\section{INTRODUCTION}

Consider a simple undirected graph $G$. To form its central graph $C(G)$, we introduce a new node on every edge in $G$ and join those nodes of $G$ that are not adjacent.The achromatic number was introduced by Harary. A proper vertex colouring is said to be achromatic if every pair of colours has at least one edge joining them . The in an achromatic colouring of $G$.

A double wheel graph $D w_{n}$ of size $n$ is composed of $2 C_{n}$ and $K_{1}$. It consists of two cycles of size $n$ where the vertices of the two cycles are connected to a central root vertex.

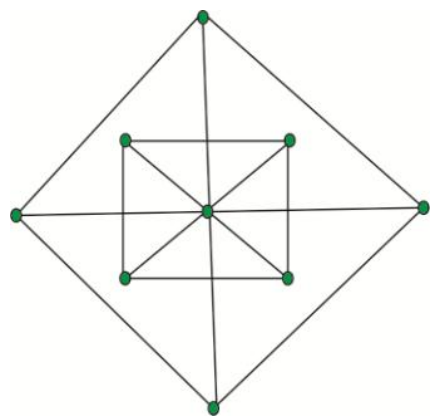

Double wheel $D w_{4}$

The wind mill graph $W m_{n}$ is obtained by joining $n$-copies of the complete graph $K_{n}$ with a vertex in common. achromatic number $\psi(G)$ is the maximum number of colours

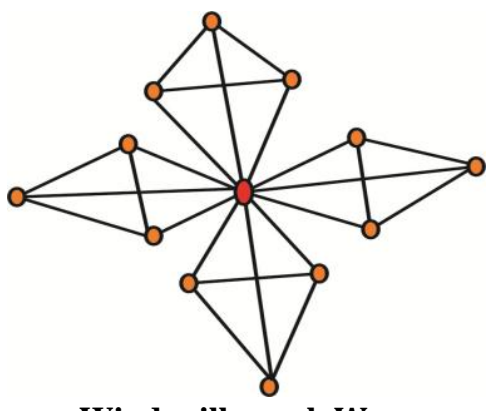

Wind mill graph $\mathrm{Wm}_{4}$

An $n$-anti prism $n \geq 3$ is a semi regular polyhedron constructed with two $n$-gons and $2 n$-triangles. It is made up of two $n$-gons on the top and bottom separated by a ribbon of $2 n$ - triangles, with the two $n$-gons being offset by one ribbon. The graph corresponding to the skeleton of an $n$-anti prism is called the $n$-anti prism graph and is denoted by $A p_{n}, n \geq 3$.

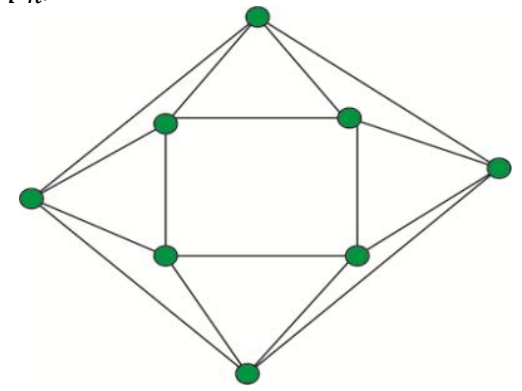

Anti prism graph $\mathrm{Ap}_{4}$

Structural properties of central graph of double wheel $\operatorname{graphD} w_{n}$

- $\quad$ The number of vertices in $D w_{n}=p=2 n+1$.

- $\quad$ The number of edges in $D w_{n}=q=4 n$.

- The maximum degree in $C\left(D w_{n}\right)=\Delta_{C\left[D w_{n}\right]}=2 n$.

- The number of vertices in $C\left(D w_{n}\right)=p_{C\left[D w_{n}\right]}=$ $6 n+1$.

- $\quad$ The number of edges in $C\left[W m_{n}\right]=q_{C\left[D W_{n}\right]}=$ $2 n^{2}+5 n$.

Theorem: 1

For a double wheel graph $D w_{n}$, the achromatic number of $C\left(D w_{n}\right)$ is $\psi\left(C\left(D w_{n}\right)\right)=2 n+1, n \geq 3$.

Proof:

A double wheel graph $D w_{n}$ of size $n$ is composed of $2 C_{n}$ and $K_{1}$. Consider the two sets of vertices

K.P.Thilagavathy, Assistant Professor, Department of Science and Humanities, Kumaraguru College of Technology, Coimbatore, Tamilnadu, India.

A.Santha, Associate Professor, Department of Science and Humanities, Kumaraguru College of Technology, Coimbatore, Tamilnadu, India.

G.S. Nandakumar, Associate Professor, Department of Computer Science and Engineering, Kumaraguru College of Technology, Coimbatore, Tamilnadu, India. 
$\left\{a_{0}, a_{1}, \ldots, a_{n}\right\}$ and $\left\{b_{1}, b_{2}, \ldots, b_{n}\right\}$. Let $a_{0}$ be the root vertex. Allocate the names $a_{0}, a_{1}, \ldots, a_{n}$ to the remaining vertices of the inner wheel and $b_{1}, b_{2}, \ldots, b_{n}$ to the vertices of the outer wheel. In $C\left(D w_{n}\right)$, for $1 \leq i \leq n-1$, let $a_{i, i+1}$ be the new vertex on the edge $a_{i}, a_{i+1}$ and let $a_{1, n}$ be the vertex on the edge joining $a_{1}, a_{n}$. Let $b_{i, i+1}$ be the newly introduced vertex on the line joining $b_{i}, b_{i+1}$ for $1 \leq i \leq n-1$. Let $a_{0, i}$ be the newly introduced vertex on the edge joining $a_{0}, a_{i}$. For $1 \leq i \leq n$, let $b_{0, i}$ be the newly introduced vertex on the edge joining $a_{0}, b_{i}$.

Let $C=\left\{C_{0}, C_{1}, C_{2}, \ldots C_{n}\right\}$ and $C^{\prime}=\left\{C^{\prime}{ }_{1}, C^{\prime}{ }_{2}, \ldots C_{n}^{\prime}\right\}$ be two colour sets. Allocate colour $C_{0}$ to the root vertex $a_{0}$. For $1 \leq i \leq$ nallocate $C_{i}$ to $a_{i}$ and $C_{i}{ }^{\prime}$ to $b_{i}$. For creating an achromatic colouring, consider the colouring method as follows:

- For $1 \leq i \leq n-1$ allocate $C_{i}$ to the vertex $a_{0, i+1}$.

- For $2 \leq i \leq n-1$ allocate the colour $C_{i}$ to the vertex $a_{i, i+1}$.

- $\quad$ Allocate the colour $C_{n}$ to the vertices $a_{0,1}$ and $a_{1,2}$.

- Allocate the colour $C_{n-1}$ to the vertex $a_{1, n}$.

- For $1 \leq i \leq n-1$ allocate $C_{i}^{\prime}$ to the vertex $b_{0, i+1}$.

- For $2 \leq i \leq n-1$ allocate the colour $C_{i}^{\prime}$ to the vertex $b_{i, i+1}$.

- $\quad$ Allocate the colour $C_{n}^{\prime}$ to the vertices $b_{0,1}$ and $b_{1,2}$.

- Allocate the colour $C_{n-1}^{\prime}$ to the vertex $b_{1, n}$.

If a new colour $C_{n+1}^{\prime}$ is given to any vertex, the other colours will not be adjacent to it. Hence the maximum number of colours for an achromatic colouring is $|C|+$ $\left|C^{\prime}\right|=2 n+1$.

\section{Example}

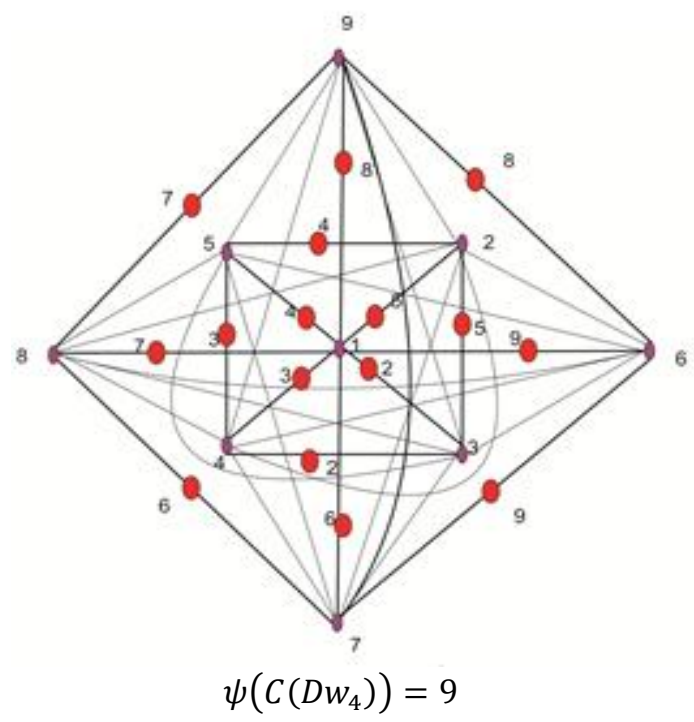

Structural properties of the central graph of windmill graph

- The number of vertices in $W m_{n}=p=n^{2}-n+1$

- $\quad$ The number of edges in $W m_{n}=q=\frac{n^{2}(n-1)}{2}$.

- The maximum degree in $W m_{n}=\Delta_{C\left[W m_{n}\right]}=$ $n(n-1)$.

- The number of vertices in $C\left[W m_{n}\right]=p_{C\left[W m_{n}\right]}=$ $\frac{n^{3}}{2}+\frac{n^{2}}{2}-n+1$.
- The number of edges in $C\left[W m_{n}\right]=q_{C\left[W m_{n}\right]}=$ $\frac{n^{3}-n^{2}-2 n+2}{2}$.

\section{OBSERVATION \& RESULTS}

For a wind mill graphWm$m_{n}$, the achromatic number of $C\left(W m_{n}\right)$ is $\psi\left(C\left(W m_{n}\right)\right)=n^{2}-n+1, n \geq 3$.

Structural properties of the central graph of Antiprism $\operatorname{graphAp}_{n}$

- $\quad$ The number of vertices in $A p_{n}=p=2 n$.

- $\quad$ The number of edges in $A p_{n}=q=4 n$.

- The maximum degree in $A p_{n}=\Delta_{C\left[A p_{n}\right]}=4$.

- The number of vertices in $C\left[A p_{n}\right]=p_{C\left[A p_{n}\right]}=6 n$.

- The number of edges in $C\left[A p_{n}\right]=q_{C\left[A p_{n}\right]}=2 n^{2}+$ $3 n$.

\section{Theorem: 3}

For an anti prism graph $A p_{n}$, the achromatic number of $C\left(A p_{n}\right)$ is $\psi\left(C\left(A p_{n}\right)\right)=2 n+1, n \geq 3$.

Proof:

Consider the two sets of vertices $U=\left\{a_{1}, \ldots, a_{n}\right\}$ and $V=\left\{b_{1}, b_{2}, \ldots, b_{n}\right\}$. Allocate the names in the set $U$ to the vertices of the inner cycle in the clock wise direction and allocate the names in the set $V$ to the vertices of the outer cycle in the same direction.

- In $C\left(A p_{n}\right)$, for let $a_{i, i+1}$ be the new vertex on the line connecting $a_{i}$ and $a_{i, i+1}, 1 \leq i \leq n-1$.

- Let $a_{1, n}$ be the vertex on the line connecting $a_{1}$ and $a_{n}$.

- Let $b_{i, i+1}$ be the newly created vertex on the line connecting $b_{i}$ and $b_{i, i+1}, 1 \leq i \leq n-1$.

- Let $b_{1, n}$ be the vertex on the line connecting $b_{1}$ and $b_{n}$

- $\quad$ For $1 \leq i \leq n$, let $a b_{i}$ be the newly created vertex on the line connecting $a_{i}$ and $b_{i}$.

- For $1 \leq i \leq n-1$, let $a_{i+1} b_{i}$ be the newly created vertex on the line connecting $a_{i+1}$ and $b_{i}$.

- Let $a_{1} b_{n}$ be the vertex on the line connecting $a_{1}$ and $b_{n}$

Let $C=\left\{C_{1}, C_{2}, \ldots C_{n}\right\}$ and $C^{\prime}=\left\{C_{1}^{\prime}, C_{2}^{\prime}, \ldots C_{n}^{\prime}, C^{\prime}{ }_{n+1}\right\}$ be two sets of colours.

For $1 \leq i \leq$ nallocate $C_{i}$ to $b_{i}$ and $C_{i}^{\prime}$ to $b_{i}$.

To make this an achromatic colouring, consider the following pattern of colouring:

- For $2 \leq i \leq n-2$ allocate $C_{i}$ to the vertex $a_{i+1, i+2}$, $C_{n-1}$ to the vertex $a_{1, n}$ and $C_{n}$ to $a_{1,2}$.

- $\quad$ For $2 \leq i \leq n-2$ allocate $C_{i}$ to the vertex $b_{i+1, i+2}$ and $C_{n-1}$ to the vertex $b_{1, n}$ and allocate $C_{n}$ to $b_{1,2}$.

- $\quad$ For $1 \leq i \leq n$ allocate the new colour $C_{n+1}^{\prime}$ to the vertex $a_{i+1} b_{i}$.

- $\quad$ For $1 \leq i \leq n-1$ allocate the new colour $C_{i+1}^{\prime}$ to the vertex $a b_{i}$ and allocate the colour $C_{1}^{\prime}$ to the vertex $a b_{n}$.

By this allotment, the maximum possible number of colours for an achromatic colouring is $|C|+\left|C^{\prime}\right|=2 n+1$.

Published By: 


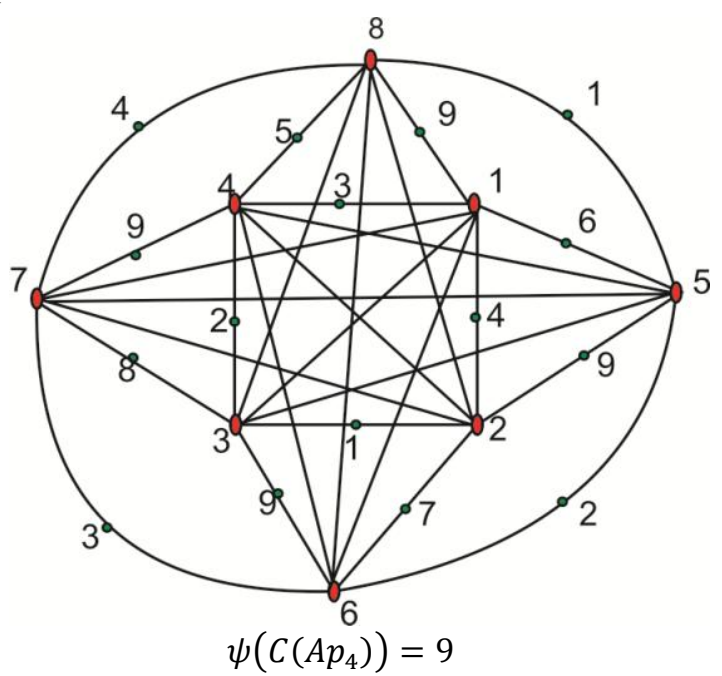

\section{REFERENCES}

1. Irving. R.W and Manlove. D.F. The b-Chromatic number of a graph. Discrete Applied Mathematics 91(1-3) 127$141,1999$.

2. Jonathan gross and Jay yellan.Handbook of graph theory CRC press, New York, 2004.

3. Marko Jakovac and Sandi Klavzar. The b-chromatic number of cubic Graphs. Graphs and Combinatorics, 26:107-118, 2010.

4. Rokad A.H. Product cordial labelling of Double wheel and Double Fan Related Graphs, Kragujevac Journal of Matematics, Volume 43(1) 7-13, 2019.

5. Thilagavathy. K.P and Santha. A. A comparative study on achromatic and b-chromatic number of certain graphs. Italian Journal of Pure and Applied Mathematics", 3944, 2018.

6. Nagaraj Balakrishnan, Reshmi S., and R. Arunkumar. "Smart real time rescue system for fishermen." Pak J Biotechnol 15, no. 1 (2018): 73-75.

7. Thilagavathy. K.P and Santha. A. The Achromatic and bChromatic Colouring of Central Graph of Book Graph and Shadow graph of Path graph.International Journal of pure and applied mathematics, 113,1-9, 2017.

8. Vernold Vivin, J. Thilagavathi, K, "On Harmonious colouring of Central Graphs", Far East. Math. Sci (FJMS), 2, pp189-197, 2006. 